\title{
The Singular Points of Analytic Functions with Finite $X$-Order Defined by Laplace-Stieltjes Transformations
}

\author{
Hong-Yan $X u^{1}$ and Zu-Xing Xuan ${ }^{2}$ \\ ${ }^{1}$ Department of Informatics and Engineering, Jingdezhen Ceramic Institute, Jingdezhen, Jiangxi 333403, China \\ ${ }^{2}$ Beijing Key Laboratory of Information Service Engineering, Department of General Education, Beijing Union University, \\ No. 97 Bei Si Huan Dong Road, Chaoyang District, Beijing 100101, China
}

Correspondence should be addressed to Zu-Xing Xuan; xuanzuxing@ss.buaa.edu.cn

Received 19 April 2014; Accepted 18 July 2014

Academic Editor: Seppo Hassi

Copyright (c) 2015 H.-Y. Xu and Z.-X. Xuan. This is an open access article distributed under the Creative Commons Attribution License, which permits unrestricted use, distribution, and reproduction in any medium, provided the original work is properly cited.

We study the singular points of analytic functions defined by Laplace-Stieltjes transformations which converge on the right half plane, by introducing the concept of $X$-order functions. We also confirm the existence of the finite $X$-order Borel points of such functions and obtained the extension of the finite $X$-order Borel point of two analytic functions defined by two Laplace-Stieltjes transformations convergent on the right half plane. The main results of this paper are improvement of some theorems given by Shang and Gao.

\section{Introduction}

For Laplace-Stieltjes transforms

$$
F(s)=\int_{0}^{+\infty} e^{-s x} d \alpha(x), \quad s=\sigma+i t
$$

where $\alpha(x)$ is a bounded variation on any interval $[0, Y](0<$ $Y<+\infty)$ and $\sigma$ and $t$ are real variables. We choose a sequence $\left\{\lambda_{n}\right\}_{n=1}^{\infty}$,

$$
0=\lambda_{1}<\lambda_{2}<\lambda_{3}<\cdots<\lambda_{n} \uparrow+\infty,
$$

which satisfies the following conditions:

$$
\begin{gathered}
\limsup _{n \rightarrow+\infty}\left(\lambda_{n+1}-\lambda_{n}\right)<+\infty, \quad \limsup _{n \rightarrow+\infty} \frac{n}{\lambda_{n}}=D<\infty \\
\limsup _{n \rightarrow+\infty} \frac{\log A_{n}^{*}}{\lambda_{n}}=0
\end{gathered}
$$

where

$$
A_{n}^{*}=\sup _{\lambda_{n}<x \leq \lambda_{n+1},-\infty<t<+\infty}\left|\int_{\lambda_{n}}^{x} e^{-i t y} d \alpha(y)\right| .
$$

Remark 1. Dirichlet series was regarded as a special example of Laplace-Stieltjes transformations; a number of articles have focused on the growth and the value distribution of analytic functions defined by Dirichlet series; see [1-3] for some recent results.

In 1963, Yu [4] proved the Valiron-Knopp-Bohr formula of the associated abscissas of bounded convergence, absolute convergence, and uniform convergence of Laplace-Stieltjes.

Theorem A. Suppose that Laplace-Stieltjes transformations (1) satisfy the first formula of (3) and $\lim \sup _{n \rightarrow+\infty}(\log n /$ $\left.\lambda_{n}\right)<+\infty$; then

$$
\limsup _{n \rightarrow+\infty} \frac{\log A_{n}^{*}}{\lambda_{n}} \leq \sigma_{u}^{F} \leq \limsup _{n \rightarrow+\infty} \frac{\log A_{n}^{*}}{\lambda_{n}}+\limsup _{n \rightarrow+\infty} \frac{\log n}{\lambda_{n}}
$$

where $\sigma_{u}^{F}$ is called the abscissa of uniform convergence of $F(s)$. 
By (3), (4), and Theorem A, one can get that $\sigma_{u}^{F}=0$; that is, $F(s)$ is analytic in the right half plane. Set

$$
\begin{gathered}
\mu(\sigma, F)=\max _{n \in N}\left\{A_{n}^{*} e^{-\lambda_{n} \sigma}\right\} \quad(\sigma>0), \\
M(\sigma, F)=\sup _{-\infty<t<+\infty}|F(\sigma+i t)|, \\
M_{u}(\sigma, F)=\sup _{0<x<+\infty,-\infty<t<+\infty}\left|\int_{0}^{x} e^{-(\sigma+i t) y} d \alpha(y)\right|, \quad(\sigma>0) .
\end{gathered}
$$

Remark 2. From (4), for any $\sigma>0$, we have

$$
\limsup _{n \rightarrow+\infty} \frac{\log A_{n}^{*}-\lambda_{n} \sigma}{\lambda_{n}}=-\sigma<0
$$

$$
\text { or } \limsup _{n \rightarrow+\infty} \log A_{n}^{*} e^{-\lambda_{n} \sigma}=-\infty \text {. }
$$

This shows that $\mu(\sigma, F)$ exists.

In the past few decades, many people studied some problems of analytic functions defined by Laplace-Stieltjes transformations and obtained a number of interesting and important results (including [5-11]). In those papers, there are about two methods to control the growth of the maximum modulus $M_{u}(\sigma, F)$ or the maximum term $\mu(\sigma, F)$ : one method is to replace the denominator in the definition of growth order by using the technique of type function $U(x)$ (see $[4,12-14]$ ) and the other method is to take multiple logarithm to $M_{u}(\sigma, F)$ or $\mu(\sigma, F)$ in the definition of growth order (see $[15,16])$. For the second method, as the logarithm function is a special function, a question rises naturally: whether we can find a relatively general function to replace the logarithm function to control the maximum growth rate.

In this paper, we investigate the above question and give a positive answer to this question. Moreover, we confirm the existence of singular points for these functions by applying the main results of our paper. To do this, we introduce a completely new technique based on the concept of $X(x)$ which is different with the type function $U(x)$ and more general than logarithm function and obtain the main theorems as follows.

Theorem 3. If the Laplace-Stieltjes transformation $F(s)$ of infinite order has finite $X$-order and the sequence (2) satisfies (3) and (4), then one has

$$
\begin{aligned}
& \limsup _{\sigma \rightarrow 0^{+}} \frac{X\left(\log ^{+} M_{u}(\sigma, F)\right)}{\log (1 / \sigma)}=\rho^{*} \\
& \Longleftrightarrow \limsup _{\sigma \rightarrow 0^{+}} \frac{X\left(\log ^{+} \mu(\sigma, F)\right)}{\log (1 / \sigma)}=\rho^{*} .
\end{aligned}
$$

Theorem 4. If the Laplace-Stieltjes transformation $F(s)$ of infinite order and the sequence (2) satisfy (3) and (4), then one has

$$
\begin{gathered}
\limsup _{\sigma \rightarrow 0^{+}} \frac{X\left(\log ^{+} M_{u}(\sigma, F)\right)}{\log (1 / \sigma)}=\rho^{*} \\
\Longleftrightarrow \limsup _{n \rightarrow \infty} \frac{X\left(\lambda_{n}\right)}{\log ^{+}\left(\lambda_{n} / \log ^{+} A_{n}^{*}\right)}=\rho^{*},
\end{gathered}
$$

where $0<\rho^{*}<\infty$.

Remark 5. The definitions of $X$-order and the function in Theorems 3 and $4 X(x)$ will be introduced in Section 2.

From Theorem 4, we further investigate the value distribution of analytic functions with finite $X$-order represented by Laplace-Stieltjes transformations convergent on the right half plane and obtain the following theorems.

Theorem 6. Suppose that the sequence (2) satisfies (3) and (4) and Laplace-Stieltjes transformation $F(s)$ is of infinite order. Let $\alpha(x)=\alpha_{1}(x)+i \alpha_{2}(x)$, where $\alpha_{1}(x)$ is a creasing function, and, for any positive number $K>0$ and $|\delta|, \alpha_{2}(x)$ satisfies

$$
\begin{gathered}
\left|\alpha_{2}(x+\delta)-\alpha_{2}(x)\right| \leq K\left|\alpha_{1}(x+\delta)-\alpha_{1}(x)\right|, \\
0 \leq x, \quad x+\delta<+\infty, \\
\limsup _{n \rightarrow \infty} \frac{X\left(\lambda_{n}\right)}{\log ^{+}\left(\lambda_{n} /\left(\log ^{+} A_{n}^{*}\right)\right)}=\rho^{*}, \quad\left(0<\rho^{*}<\infty\right),
\end{gathered}
$$

and then $s=0$ is the $X$-point of $F(s)$ with $X$-order $\varrho \geq \rho^{*}$; that is, for any $\eta>0$, the inequality

$$
\limsup _{\sigma \rightarrow 0^{+}} \frac{X(\bar{n}(\sigma, 0, \eta, F=a))}{\log (1 / \sigma)}=\varrho \geq \rho^{*}
$$

holds for any $a \in \mathbb{C}$, except for one exception, where $\bar{n}(\sigma, 0$, $\eta, F=a)$ is the counting function of distinct zero of the function $F(s)-a$ in the strip $\{s: \mathfrak{R}(s)>\sigma,|\mathfrak{\Im}(s)|<\eta\}$.

Theorem 7. Suppose that the sequence (2) satisfies (3) and (4) and Laplace-Stieltjes transformation $F(s)$ is of infinite order. Let $\alpha(x)=\int_{0}^{x} r(y) e^{i t_{0} y} d y$, where $r(y)$ is continuous function on $y \in[0,+\infty), r(y) \geq 0, t_{0}$ is a positive real number, and

$$
\limsup _{n \rightarrow \infty} \frac{X\left(\lambda_{n}\right)}{\log ^{+}\left(\lambda_{n} / \log ^{+} A_{n}^{*}\right)}=\rho^{*}, \quad\left(0<\rho^{*}<\infty\right) ;
$$

then $s=i t_{0}$ is the $X$-point of $F(s)$ with $X$-order $\varrho \geq \rho^{*}$; that is, for any $\eta>0$, the inequality

$$
\limsup _{\sigma \rightarrow 0^{+}} \frac{X\left(\bar{n}\left(\sigma, i t_{0}, \eta, F=a\right)\right)}{\log (1 / \sigma)}=\varrho \geq \rho^{*}
$$

holds for any $a \in \mathbb{C}$, except for one exception, where $\bar{n}\left(\sigma, i t_{0}\right.$, $\eta, F=a)$ is the counting function of distinct zeros of the function $F(s)-a$ in the strip $\left\{s: \mathfrak{R}(s)>\sigma,\left|\mathfrak{\Im}(s)-t_{0}\right|<\eta\right\}$. 
The structure of this paper is as follows. In Section 2, we introduce the concept of $X$-order and give the proofs of Theorems 3 and 4. Section 3 is devoted to proving Theorems 6 and 7.

Remark 8. From Theorems 3-7, we assume that the $X$-order $\rho *$ of $F(s)$ is finite; that is, $0<\rho<\infty$. For $\rho^{*}=+\infty$, we have studied the value distribution of analytic functions defined by Laplace-Stieltjes transformations which converge on the right half plane and obtained some results (see [17]).

\section{Proofs of Theorems 3 and 4}

We first introduce the concept of $X$-order of such functions as follows.

Definition 9 (see [18]). If the Laplace-Stieltjes transform $F(s)$ satisfies $\sigma_{u}^{F}=0$ (the sequence (2) satisfies (3) and (4)) and

$$
\limsup _{\sigma \rightarrow 0^{+}} \frac{\log ^{+} \log ^{+} M_{u}(\sigma, F)}{\log (1 / \sigma)}=\infty
$$

then $F(s)$ is called a Laplace-Stieltjes transform of infinite order.

To control the growth of the molecule $M_{u}(\sigma, F)$ or $\mu(\sigma, F)$ in the definition of order, many mathematicians proposed the type functions $U(x)$ to enlarge the growth of the denominator $\log (1 / \sigma)$ or $-\sigma$ (see $[4,6,11,13,14])$. In this paper, we will investigate the growth of Laplace-Stieltjes transform of infinite order by using a class of functions to reduce the growth of $M_{u}(\sigma, F)$ or $\mu(\sigma, F)$ which is different with the previous form. Thus, we should give the definition of the new function as follows.

Let $\mathfrak{F}$ be the class of all functions $X$ which satisfies the following conditions:

(i) $X(x)$ is defined on $[a,+\infty), a>0$, is positive, strictly increasing, and differential, and tends to $+\infty$ as $x \rightarrow$ $+\infty$;

(ii) $x X^{\prime}(x)=o(1)$ as $x \rightarrow+\infty$.

Definition 10. If the Laplace-Stieltjes transformation $F(s)$ of infinite order satisfies

$$
\limsup _{\sigma \rightarrow 0^{+}} \frac{X\left(\log M_{u}(\sigma, F)\right)}{\log (1 / \sigma)}=\rho^{*},
$$

where $X(x) \in \mathfrak{F}$, then $\rho^{*}$ is called the $X$-order of the LaplaceStieltjes transform $F(s)$.

Remark 11. In particular, if we take $X(x)=\log _{p} x, p \geq 2, p \in$ $N_{+}$, where $\log _{1} x=\log x$ and $\log _{p} x=\log \left(\log _{p-1} x\right), X$-order is $p$-order of Laplace-Stieltjes transformations with infinite order.

Remark 12. In addition, $X$-order is more precise than $p$ order. In fact, for $p(\geq 2)$ being a positive integer, we can find out function $X(x) \in \mathfrak{F}$ and a positive real function $M(x)$ satisfying

$$
\begin{gathered}
\limsup _{x \rightarrow \infty} \frac{X(M(x))}{\log x}=A, \quad(0<A<\infty), \\
\limsup _{x \rightarrow \infty} \frac{\log _{p} M(x)}{\log x}=\infty, \quad \limsup _{x \rightarrow \infty} \frac{\log _{p+1} M(x)}{\log x}=0 .
\end{gathered}
$$

For example, letting $M(x)=\exp _{p}\left\{(t \log x)^{1 / d}\right\}, X(x)=$ $\left(\log _{p} x\right)^{d}$, where $t$ is a finite positive real constant and $0<d<$ 1.

The following lemma is very important to study the growth of analytic functions represented by Laplace-Stieltjes transforms convergent on the right half plane, which show the relation between $M_{u}(\sigma, F)$ and $\mu(\sigma, F)$ of such functions.

Lemma 13 (see $[7,11])$. If the abscissa $\sigma_{u}^{F}=0$ of uniform convergence of Laplace-Stieltjes transformation and the sequence (2) satisfy (3), then, for any given $\varepsilon \in(0,1)$ and for $\sigma(>0)$ sufficiently reaching 0 , one has

$$
\frac{1}{3} \mu(\sigma, F) \leq M_{u}(\sigma, F) \leq K(\varepsilon) \mu((1-\varepsilon) \sigma, F) \frac{1}{\sigma},
$$

where $K(\varepsilon)$ is a constant depending on $\varepsilon$, (3), and

$$
\log ^{+} x= \begin{cases}\log x & x \geq 1 \\ 0 & x<1\end{cases}
$$

2.1. The Proof of Theorem 3. Firstly, for any constant $K_{1}$, we will prove that

$$
\limsup _{\sigma \rightarrow 0^{+}} \frac{X\left(K_{1} \log \mu(\sigma, F)\right)}{\log (1 / \sigma)}=\limsup _{\sigma \rightarrow 0^{+}} \frac{X(\log \mu(\sigma, F))}{\log (1 / \sigma)} .
$$

Without loss of generality, we suppose that $K_{1}>1$. From the Laplace-Stieltjes transformation $F(s)$ with infinite order and Lemma 13, we have $\lim _{\sigma \rightarrow 0^{+}} \mu(\sigma, F)=\infty$. Then, from the Cauchy mean value theorem, there exists $\eta(\log \mu(\sigma, F)<$ $\left.\eta<K_{1} \log \mu(\sigma, F)\right)$ satisfying

$$
\frac{X\left(K_{1} \log \mu(\sigma, F)\right)-X(\log \mu(\sigma, F))}{\log \left(K_{1} \log \mu(\sigma, F)\right)-\log \log \mu(\sigma, F)}=\frac{X^{\prime}(\eta)}{(\log \xi)^{\prime}}=\eta X^{\prime}(\eta) ;
$$

that is,

$$
X\left(K_{1} \log \mu(\sigma, F)\right)=X(\log \mu(\sigma, F))+\log K_{1} \eta X^{\prime}(\eta) .
$$

From $x X^{\prime}(x)=o(1)$ as $x \rightarrow+\infty$ and the above equality, we can easily get (20).

Thus, from (20) and Lemma 13, we can prove the conclusion of Theorem 3 easily. 
2.2. The Proof of Theorem 4. $(\Leftarrow)$ : Suppose that

$$
\limsup _{n \rightarrow \infty} \frac{X\left(\lambda_{n}\right)}{\log ^{+}\left(\lambda_{n} / \log A_{n}^{*}\right)}=\rho^{*} .
$$

We consider two steps in this case as follows.

Step 1. We will prove that $\lim \sup _{\sigma \rightarrow 0^{+}}\left(X\left(\log ^{+} M_{u}(\sigma, F)\right) /\right.$ $\log (1 / \sigma)) \geq \rho^{*}$. From $(23)$ and $0<\rho^{*}<\infty$, there exists a positive sequence $\left\{n_{k}\right\}$; for any $\varepsilon\left(0<\varepsilon<\rho^{*}\right)$,

$$
\log A_{n_{k}}^{*}>\lambda_{n_{k}} \exp \left\{-\frac{1}{\rho^{*}-\varepsilon} X\left(\lambda_{n_{k}}\right)\right\}, \quad k=1,2,3, \ldots
$$

From (18) and (23), we have

$$
\begin{aligned}
\log M_{u}(\sigma, F) \geq & \log A_{n_{k}}^{*}-\lambda_{n_{k}} \sigma+O(1) \\
> & \lambda_{n_{k}} \exp \left\{-\frac{1}{\rho^{*}-\varepsilon} X\left(\lambda_{n_{k}}\right)\right\} \\
& -\sigma \lambda_{n_{k}}+O(1)
\end{aligned}
$$

Setting $\sigma=\sigma_{k}$ and taking $\sigma_{k}=(1 / 2) \exp \left\{\left(-1 /\left(\rho^{*}-\right.\right.\right.$ $\left.\varepsilon)) X\left(\lambda_{n_{k}}\right)\right\},(k=1,2,3, \ldots)$, then, from (24) and (25), we have

$$
\log M_{u}\left(\sigma_{k}, F\right)>\frac{1}{2} \lambda_{n_{k}} \exp \left\{-\frac{1}{\rho^{*}-\varepsilon} X\left(\lambda_{n_{k}}\right)\right\}(1+o(1))
$$

that is,

$$
(1+o(1)) \frac{1}{\sigma_{k}} \log M_{u}\left(\sigma_{k}, F\right)>\lambda_{n_{k}}
$$

From $\sigma_{k}=(1 / 2) \exp \left\{\left(-1 /\left(\rho^{*}-\varepsilon\right)\right) X\left(\lambda_{n_{k}}\right)\right\}$, we have

$$
X\left(\lambda_{n_{k}}\right)=\left(\rho^{*}-\varepsilon\right) \log \frac{1}{2 \sigma_{k}} .
$$

Thus, from (27), (28) and $X(x)$ being strictly increasing function, we have

$$
X\left((1+o(1)) \frac{1}{\sigma_{k}} \log M_{u}\left(\sigma_{k}, F\right)\right)>\left(\rho^{*}-\varepsilon\right) \log \frac{1}{2 \sigma_{k}} .
$$

Since $\sigma>0$, from the Cauchy mean value theorem, there exists $\zeta\left(\log M_{u}\left(\sigma_{k}, F\right)<\zeta<(1+o(1))\left(1 / \sigma_{k}\right) \log M_{u}\left(\sigma_{k}, F\right)\right)$ satisfying

$$
\begin{aligned}
& \frac{X\left((1+o(1))\left(1 / \sigma_{k}\right) \log M_{u}\left(\sigma_{k}, F\right)\right)-X\left(\log M_{u}\left(\sigma_{k}, F\right)\right)}{\log \left((1+o(1))\left(1 / \sigma_{k}\right) \log M_{u}\left(\sigma_{k}, F\right)\right)-\log \log M_{u}\left(\sigma_{k}, F\right)} \\
& \quad=\frac{X^{\prime}(\zeta)}{(\log \zeta)^{\prime}}=\zeta X^{\prime}(\zeta) ;
\end{aligned}
$$

that is,

$$
\begin{aligned}
X( & \left.(1+o(1)) \frac{1}{\sigma_{k}} \log M_{u}\left(\sigma_{k}, F\right)\right) \\
= & X\left(\log M_{u}\left(\sigma_{k}, F\right)\right) \\
& +\log \left[(1+o(1)) \frac{1}{\sigma_{k}}\right] \zeta X^{\prime}(\zeta) .
\end{aligned}
$$

Since $x X^{\prime}(x)=o(1)$ as $x \rightarrow+\infty$, from (23) and (31), we can get

$$
\limsup _{\sigma \rightarrow 0^{+}} \frac{X\left(\log ^{+} M_{u}(\sigma, F)\right)}{\log (1 / \sigma)} \geq \rho^{*}
$$

Step 2. We will prove that $\lim \sup _{\sigma \rightarrow 0^{+}}\left(X\left(\log ^{+} M_{u}(\sigma, F)\right) /\right.$ $\log (1 / \sigma)) \leq \rho^{*}$.

From $0<\rho^{*}<\infty$ and (23), there exists a positive integer $n_{0}$; for any $\varepsilon(>0)$, we have

$$
\log A_{n}^{*}<\lambda_{n} \exp \left(-\frac{1}{\rho^{*}+\varepsilon} X\left(\lambda_{n}\right)\right), \quad n>n_{0} .
$$

From (3), for any $\varepsilon^{\prime}(>0)$ and the above $\varepsilon$, there exists a positive integer $N_{1}\left(>n_{0}\right)$ satisfying

$$
\begin{array}{r}
N_{1} \leq\left(D+\varepsilon^{\prime}\right) W\left(\left(\rho^{*}+\varepsilon\right) \log \frac{2}{\sigma}\right)<N_{1}+1, \\
\text { as } \sigma \longrightarrow 0^{+},
\end{array}
$$

where $W(x)$ and $X(x)$ are two reciprocally inverse functions. Set $\lambda_{n}=W\left(\left(\rho^{*}+\varepsilon\right) \log (2 / \sigma)\right)$, and then

$$
\begin{gathered}
\sum_{n=N_{1}+1}^{\infty} \exp \left(\lambda_{n} \exp \left(\frac{-1}{\rho^{*}+\varepsilon} X\left(\lambda_{n}\right)\right)-\lambda_{n} \sigma\right) \\
<\exp \left(-\frac{\lambda_{n} \sigma}{2}\right) \\
\quad<\sum_{n=N_{1}+1}^{\infty} \exp \left(\frac{-n \sigma}{2\left(D+\varepsilon^{\prime}\right)}\right) \\
=\frac{\exp \left(\left(-\sigma\left(N_{1}+1\right)\right) /\left(2\left(D+\varepsilon^{\prime}\right)\right)\right)}{1-\exp \left(-\sigma /\left(2\left(D+\varepsilon^{\prime}\right)\right)\right)} .
\end{gathered}
$$

From the above inequality and (34), we have

$$
\begin{aligned}
\log \frac{\exp \left(\left(-\sigma\left(N_{1}+1\right)\right) /\left(2\left(D+\varepsilon^{\prime}\right)\right)\right)}{1-\exp \left(-\sigma / 2\left(D+\varepsilon^{\prime}\right)\right)} \\
\quad=\frac{-\sigma\left(N_{1}+1\right)}{2\left(D+\varepsilon^{\prime}\right)}+\log \frac{2}{\sigma}+O(1) \\
\quad<-\frac{\sigma}{2} W\left(\left(\rho^{*}+\varepsilon\right) \log \frac{2}{\sigma}\right)+\log \frac{2}{\sigma}+O(1), \quad \sigma \rightarrow 0^{+} .
\end{aligned}
$$


Next, we will prove that

$$
\begin{aligned}
\log \frac{\exp \left(\left(-\sigma\left(N_{1}+1\right)\right) /\left(2\left(D+\varepsilon^{\prime}\right)\right)\right)}{1-\exp \left(-\sigma / 2\left(D+\varepsilon^{\prime}\right)\right)} & \longrightarrow-\infty, \\
\sigma & \longrightarrow 0^{+} .
\end{aligned}
$$

By using the same argument as in (31), we can get that

$$
\begin{array}{r}
X\left(\left(\frac{2}{\sigma}\right)^{2} \log \frac{2}{\sigma}\right)=X\left(\log \frac{2}{\sigma}\right)+o(1) \log \left(\frac{2}{\sigma}\right)^{2}, \\
\text { as } \sigma \longrightarrow 0^{+} .
\end{array}
$$

Since $F(s)$ is infinite order and finite $X$-order, from the definition of $X(x)$, we can get the following equality easily:

$$
\limsup _{x \rightarrow \infty} \frac{X(x)}{x}=0 .
$$

From (38), (39) and $0<\rho^{*}<\infty$, we have

$$
\begin{aligned}
\left(\rho^{*}+\varepsilon\right) \log \frac{2}{\sigma} & >X\left(\log \frac{2}{\sigma}\right)+o(1) \log \left(\frac{2}{\sigma}\right)^{2} \\
& =X\left(\left(\frac{2}{\sigma}\right)^{2} \log \frac{2}{\sigma}\right) .
\end{aligned}
$$

Thus, from (40), we can deduce

$$
\begin{aligned}
\limsup _{\sigma \rightarrow 0^{+}} \frac{(-\sigma / 2) W\left(\left(\rho^{*}+\varepsilon\right) \log (2 / \sigma)\right)}{\log (2 / \sigma)} \\
\quad=\limsup _{\sigma \rightarrow 0^{+}}-\frac{2}{\sigma} \longrightarrow-\infty .
\end{aligned}
$$

From (36), we can get that

$$
\begin{aligned}
\log \frac{\exp \left(\left(-\sigma\left(N_{1}+1\right)\right) /\left(2\left(D+\varepsilon^{\prime}\right)\right)\right)}{1-\exp \left(-\sigma / 2\left(D+\varepsilon^{\prime}\right)\right)} & \longrightarrow-\infty \\
& \sigma \longrightarrow 0^{+}
\end{aligned}
$$

That is,

$$
\frac{\exp \left(\left(-\sigma\left(N_{1}+1\right)\right) /\left(2\left(D+\varepsilon^{\prime}\right)\right)\right)}{1-\exp \left(-\sigma /\left(2\left(D+\varepsilon^{\prime}\right)\right)\right)} \longrightarrow 0, \quad \sigma \longrightarrow 0^{+}
$$

Hence, we have

$$
\begin{array}{r}
\sum_{n=N_{1}+1}^{\infty} \exp \left(\lambda_{n} \exp \left(-\frac{1}{\rho^{*}+\varepsilon} X\left(\lambda_{n}\right)\right)-\lambda_{n} \sigma\right) \\
\longrightarrow 0, \\
\sigma \longrightarrow 0^{+} .
\end{array}
$$

Since $X \in \mathfrak{F}$ and $x \beta^{\prime}(x) \rightarrow 0$ as $x \rightarrow \infty$, then there exists a positive integer $N_{2}\left(N_{2}>n_{0}\right)$ satisfying

$$
\frac{1}{2}<1-\frac{1}{\rho^{*}+\varepsilon} \lambda_{n} X^{\prime}\left(\lambda_{n}\right)<2, \quad n>N_{2} .
$$

Take

$$
\begin{array}{r}
\lambda_{n}=W\left(-\left(\rho^{*}+\varepsilon\right) \log \left(\sigma\left(1-\frac{1}{\rho^{*}+\varepsilon} \lambda_{n} X^{\prime}\left(\lambda_{n}\right)\right)^{-1}\right)\right), \\
n>N_{2} .
\end{array}
$$

From (45) and $W(x)$ being a strictly increasing function, we can get that $N_{2}<N_{1}$. Then, from (33), (45), (47), and $\sigma>0$, we have

$$
\begin{aligned}
& \max _{N_{2} \leq n \leq N_{1}}\left\{A_{n}^{*} \exp \left(-\sigma \lambda_{n}\right)\right\} \\
& \quad \leq \exp \left\{\lambda_{n} \exp \left(\frac{-1}{\rho^{*}+\varepsilon} X\left(\lambda_{n}\right)\right)-\sigma \lambda_{n}\right\} \\
& \quad \leq \exp \left\{\lambda_{n}\left[\sigma\left(1-\frac{1}{\rho^{*}+\varepsilon} \lambda_{n} X^{\prime}\left(\lambda_{n}\right)\right)^{-1}-\sigma\right]\right\} \\
& \quad \leq \exp \left\{-\lambda_{n} \sigma\left[1-\left(1-\frac{1}{\rho^{*}+\varepsilon} \lambda_{n} X^{\prime}\left(\lambda_{n}\right)\right)^{-1}\right]\right\} \\
& \quad \leq \exp \left\{\sigma \lambda_{n}\right\} \leq \exp \left\{\sigma W\left(\left(\rho^{*}+\varepsilon\right) \log \frac{2}{\sigma}\right)\right\} .
\end{aligned}
$$

For any $\sigma>0$ and any $t \in R$, we have

$$
\begin{aligned}
\left|\int_{0}^{x} e^{-(\sigma+i t) y} d \alpha(y)\right| \leq & \sum_{k=1}^{n-1} A_{k}^{*}\left(e^{-\lambda_{k+1} \sigma}+\left|e^{-\lambda_{k+1} \sigma}-e^{-\lambda_{k} \sigma}\right|\right) \\
& +A_{n}^{*}\left(e^{-\sigma x}+\left|e^{-\sigma x}-e^{-\lambda_{n} \sigma}\right|\right) \\
\leq & \sum_{k=1}^{n} A_{k}^{*} e^{-\lambda_{k} \sigma} .
\end{aligned}
$$

Thus, from (33), (34), (45)-(47), and the above inequality, we have

$$
\begin{aligned}
M_{u}(\sigma, F) \leq & \sum_{n=1}^{\infty} A_{n}^{*} e^{-\lambda_{n} \sigma} \\
\leq & \left(\sum_{n=1}^{N_{2}}+\sum_{n=N_{2}+1}^{N_{1}}+\sum_{n=N_{1}+1}^{\infty}\right) A_{n}^{*} \exp \left(-\sigma \lambda_{n}\right) \\
\leq & H\left(N_{2}\right)+\left(D+\varepsilon^{\prime}\right) W\left(\left(\rho^{*}+\varepsilon\right) \log \frac{2}{\sigma}\right) \\
& \times \exp \left\{\sigma W\left(\left(\rho^{*}+\varepsilon\right) \log \frac{2}{\sigma}\right)\right\}+o(1) \\
& \quad \sigma \longrightarrow 0^{+}
\end{aligned}
$$

where $H\left(N_{2}\right)$ is the sum of finite items of the series $\sum_{n=1}^{\infty} A_{n}^{*} e^{-\lambda_{n} \sigma}$. Hence, from the above inequality, we have

$$
\begin{array}{r}
\log M_{u}(\sigma, F) \leq\left(\sigma W\left(\left(\rho^{*}+\varepsilon\right) \log \frac{2}{\sigma}\right)\right)(1+o(1)), \\
\sigma \longrightarrow 0^{+} ;
\end{array}
$$


that is,

$$
O(1) \frac{1}{\sigma} \log M_{u}(\sigma, F) \leq W\left(\left(\rho^{*}+\varepsilon\right) \log \frac{2}{\sigma}\right), \quad \sigma \longrightarrow 0^{+}
$$

Then, by using the same argument as in (31), we can deduce

$$
\limsup _{\sigma \rightarrow 0^{+}} \frac{X\left(\log ^{+} M_{\mathcal{u}}(\sigma, F)\right)}{\log (1 / \sigma)} \leq \rho^{*}
$$

From Steps 1 and 2, the sufficiency of the theorem is completed.

By using the same argument as in the above proof of the theorem, we can prove the necessity of the theorem easily.

Thus, we complete the proof of Theorem 4 .

\section{Proofs of Theorems 6 and 7}

Similar to the definition of $X$-order of Laplace-Stieltjes transformations in the right half plane, we will give the definition of $X$-order of Laplace-Stieltjes transformations in the level half-strip as follows.

Definition 14. Let $F(s)$ be the analytic function with infinite order represented by Laplace-Stieltjes transformations convergent on the right half plane; set $S\left(t_{0}, l\right)=\{\sigma+i t: \sigma>$ $\left.0,\left|t-t_{0}\right| \leq l\right\}$, where $t_{0}$ is a real number and $l$ is a positive number. Let $X \in \mathfrak{F}$ and

$$
\tau_{S}^{X}=\limsup _{\sigma \rightarrow 0^{+}} \frac{X\left(\log ^{+} M_{S}(\sigma, F)\right)}{\log (1 / \sigma)}
$$

where $M_{S}(\sigma, F)=\sup _{\left|t-t_{0}\right| \leq l}|F(\sigma+i t)|$; then $\tau_{S}^{X}$ is called the $X$-order of $F(S)$ in the level half-strip $S\left(t_{0}, l\right)$.

To prove Theorems 6 and 7, we need some lemmas as follows.

Lemma 15. If the Laplace-Stieltjes transformation $F(s)$ of infinite order and the sequence (2) satisfy (3) and (4) and $\alpha(x)=\alpha_{1}(x)+i \alpha_{2}(x)$, where $\alpha_{1}(x)$ is a creasing function, and, for any positive number $K>0$ and $|\delta|, \alpha_{2}(x)$ satisfies

$$
\begin{array}{r}
\left|\alpha_{2}(x+\delta)-\alpha_{2}(x)\right| \leq K\left|\alpha_{1}(x+\delta)-\alpha_{1}(x)\right|, \\
0 \leq x, \quad x+\delta<+\infty
\end{array}
$$

then, for any $\varepsilon>0$, one has

$$
\begin{aligned}
\rho^{*} & =\limsup _{\sigma \rightarrow 0^{+}} \frac{X\left(\log ^{+} M_{u}(\sigma, F)\right)}{\log (1 / \sigma)} \\
& =\limsup _{\sigma \rightarrow 0^{+}} \frac{X\left(\log ^{+} M_{S_{\varepsilon}}(\sigma, F)\right)}{\log (1 / \sigma)}=\tau_{S_{\varepsilon}} .
\end{aligned}
$$

Proof. We will prove this lemma by using the similar argument as in [11]. From the assumptions of Lemma 15, for any $0<x \leq \infty$,

$$
\begin{aligned}
M_{S_{\varepsilon}}(\sigma, F) & \geq\left|\int_{0}^{\infty} e^{-\sigma y} d \alpha(y)\right| \\
& \geq \int_{0}^{\infty} e^{-\sigma y} d \alpha_{1}(y) \\
& \geq \frac{1}{K+1} \int_{0}^{\infty} e^{-\sigma y}\left|d \alpha_{1}(y)\right| \\
& \geq \frac{1}{K+1} \int_{0}^{x} e^{-\sigma y}\left|d \alpha_{1}(y)\right| \\
& \geq \frac{1}{K+1}\left|\int_{0}^{\infty} e^{-(\sigma+i t) y} d \alpha(y)\right|
\end{aligned}
$$

Thus, we have

$$
M(\sigma, F) \geq M_{S_{\varepsilon}}(\sigma, F) \geq \frac{1}{K+1} M_{u}(\sigma, F) \geq \frac{1}{K+1} M(\sigma, F) .
$$

Since $F(s)$ is an analytic function with infinite order, from the above inequality and the definition of $X$-order, we can get the conclusion of Lemma 15 easily.

Lemma 16 (see [11, Lemma 2.4]). Let

$$
z=\frac{1-\sinh s}{1+\sinh s}, \quad s \in B=\left\{s: \Re(s)>0,|\mathfrak{I}(s)|<\frac{\pi}{2}\right\} .
$$

Then, one has the following:

(i) this mapping maps the horizontal half-strip $B$ to the unit disc $\{z:|z|<1\}$, and its inverse mapping is

$$
s=\Psi(z)=\sinh ^{-1} \frac{1-z}{1+z}
$$

(ii) $\min _{0 \leq \theta \leq 2 \pi} \mathfrak{R}\left[\Psi\left(r e^{i \theta}\right)\right] \geq \Psi(r),(0<r<1)$;

(iii) $\max _{0 \leq \theta \leq \pi / 4} \mathfrak{R}\left[\Psi\left(r e^{i \theta}\right)\right] \leq \Psi\left(r^{2}\right),(0<r<1)$;

(iv) $\Psi(\{z:|z|<r\}) \subseteq\{s: \mathfrak{R}(s)>\Psi(r),|\mathfrak{I}(s)|<\pi / 2\}$, $(0<r<1)$.

Definition 17 (see $[19,20])$. Let $f$ be a meromorphic function in $\mathbb{D}$ and $\lim _{r \rightarrow 1^{-}} T(r, f)=\infty$. Then

$$
D(f):=\limsup _{r \rightarrow 1^{-}} \frac{T(r, f)}{-\log (1-r)}
$$

is called the (upper) index of inadmissibility of $f$. If $D(f)<$ $\infty, f$ is called nonadmissible; if $D(f)=\infty, f$ is called admissible.

Lemma 18 (see [20, Theorem 2]). Let $f$ be a meromorphic function in $\mathbb{D}$ and $\lim _{r \rightarrow 1^{-}} T(r, f)=\infty$, let $q$ be a positive 
integer, and let $a_{1}, a_{2}, \ldots, a_{q}$ be pairwise distinct complex numbers. Then, for $r \rightarrow 1^{-}, r \notin E$,

$$
(q-2) T(r, f) \leq \sum_{j=1}^{q} \bar{N}\left(r, \frac{1}{f-a_{j}}\right)+\log \frac{1}{1-r}+S(r, f) .
$$

Remark 19. We can see that $S(r, f)=o(\log (1 /(1-r)))$ holds in Lemma 18 without a possible exception set when $0<D(f)<$ $\infty$.

Remark 20. The term $\log (1 /(1-r))$ in Lemma 18 can enter the remainder $S(r, f)=o(T(r, f))$ when the function $f$ satisfies $D(f)=\infty$.

From Lemmas 3.3 and 3.4 in [21], we can get the following result for nonadmissible functions in the unit disc which is used in this paper.

Lemma 21 (see [19, page $282(1.8)]$ ). Let $h$ be analytic in the disc $|z|=r<1$; then

$$
T(r, h) \leq \log M(r, h) \leq \frac{1+r}{1-r} T(r, h),
$$

where $M(r, h)$ is the maximum modulus of $h$ in the disc $|z|=$ $r<1$.

3.1. The Proof of Theorem 6. From the sequence (2) satisfying (3) and (4), Laplace-Stieltjes transformation $F(s)$ of infinite order, and $\lim \sup _{n \rightarrow \infty}\left(X\left(\lambda_{n}\right) / \log ^{+}\left(\lambda_{n} /\left(\log ^{+} A_{n}^{*}\right)\right)\right)=\rho^{*}$, $\left(0<\rho^{*}<\infty\right)$, from Theorem 4 , we have

$$
\limsup _{\sigma \rightarrow 0^{+}} \frac{X\left(\log ^{+} M_{u}(\sigma, F)\right)}{\log (1 / \sigma)}=\rho^{*},
$$

and, from Lemma 15 and (63), for any $\varepsilon>0$, we have

$$
\limsup _{\sigma \rightarrow 0^{+}} \frac{X\left(\log ^{+} M_{S_{\varepsilon}}(\sigma, F)\right)}{\log (1 / \sigma)}=\rho^{*} .
$$

Thus, we can get

$$
\limsup _{\sigma \rightarrow 0^{+}} \frac{X\left(\log M\left(\sigma, F, S_{\varepsilon}\right)\right)}{\log (1 / \sigma)}=\rho^{*},
$$

where $S_{\varepsilon}=\{s: \Re(s)>0,|\Im(s)| \leq \varepsilon\}$ and $M\left(\sigma, F, S_{\varepsilon}\right)=$ $\sup \left\{|F(s)|: \mathfrak{R}(s) \geq \sigma, s \in S_{\varepsilon}\right\}$.

Set $g(z)=F((2 \varepsilon / \pi) \Psi(z))$, where $\Psi(z)$ is stated as in Lemma 16; then, from Lemma 16 , we have that $g(z)$ is analytic in the unit disc $|z|<1$ and satisfies

$$
M\left(\frac{2 \varepsilon}{\pi} \Psi\left(r^{2}\right), F, S_{\varepsilon_{1}}\right) \leq M(r, g) \leq M\left(\frac{2 \varepsilon}{\pi} \Psi(r), F, S_{\varepsilon}\right),
$$

where $0<\varepsilon_{1}<\varepsilon$. Therefore, from (66) and Lemma 16, we have

$$
\begin{gathered}
\limsup _{r \rightarrow 1^{-}} \frac{\log ^{+} \log ^{+} M(r, g)}{\log (1 /(1-r))}=\infty, \\
\limsup _{r \rightarrow 1^{-}} \frac{X\left(\log ^{+} M(r, g)\right)}{\log (1 /(1-r))}=\rho^{*} .
\end{gathered}
$$

From Lemma 21, by using the same argument as in (31), we can get

$$
X\left(\frac{1+r}{1-r} T(r, g)\right)=X(T(r, g))+o(1) \log \frac{1+r}{1-r}
$$

From (67), (68), and Lemma 21, we can get that $g$ is an admissible function in $|z|<1$ and

$$
\limsup _{r \rightarrow 1^{-}} \frac{X(T(r, g))}{\log (1 /(1-r))}=\rho^{*}
$$

Then, from Lemma 18, we can get that there at most exists one except value $a$ satisfying

$$
\limsup _{r \rightarrow 1^{-}} \frac{X(\bar{n}(r, g=a))}{\log (1 /(1-r))}=\varrho \geq \rho^{*}
$$

that is, for any $\eta>0$, the inequality

$$
\limsup _{\sigma \rightarrow 0^{+}} \frac{X(\bar{n}(\sigma, 0, \eta, F=a))}{\log (1 / \sigma)}=\varrho \geq \rho^{*}
$$

holds for any $a \in \mathbb{C}$, except for one exception, where $n(\sigma, 0, \eta, F=a)$ is the counting function of zeros of the function $F(s)-a$ in the strip $\{s: \mathfrak{R}(s)>\sigma,|\mathfrak{J}(s)|<\eta\}$.

Thus, we complete the proof of Theorem 6 .

3.2. The Proof of Theorem 7. Since $r(y)$ is a continuous function on $y \in[0,+\infty)$, then we can get that $\alpha(x)=$ $\int_{0}^{x} r(y) e^{i t_{0} y} d y$ is a function of bounded variation on $x \in$ $[0, X](0<X<\infty)$. Set

$$
\begin{array}{r}
\widehat{S}_{\varepsilon}=\left\{s: \Re(s)>0,\left|\mathfrak{I}(s)-t_{0}\right| \leq \varepsilon\right\}, \\
M_{\widehat{S}_{\varepsilon}}(\sigma, F)=\sup _{\left|t-t_{0}\right| \leq \varepsilon}|F(\sigma+i t)| .
\end{array}
$$

From the assumptions of Theorem 7 , for any real number $x(0<x \leq \infty)$, we have

$$
\begin{aligned}
M_{\widehat{\mathcal{S}}_{\varepsilon}}(\sigma, F) & =\sup _{\left|t-t_{0}\right| \leq \varepsilon}|F(\sigma+i t)| \\
& =\sup _{\left|t-t_{0}\right| \leq \varepsilon}\left|\int_{0}^{\infty} e^{-s y} r(y) e^{i t_{0} y} d y\right| \\
& \geq \int_{0}^{\infty} e^{-\sigma y} r(y) d y \geq \int_{0}^{x} e^{-\sigma y} r(y) d y \\
& \geq\left|\int_{0}^{x} e^{-s y} r(y) e^{i t_{0} y} d y\right|=\left|\int_{0}^{\infty} e^{-s y} d \alpha(y)\right| ;
\end{aligned}
$$

that is,

$$
M(\sigma, F) \leq M_{u}(\sigma, F) \leq M_{\widehat{\mathcal{S}}_{\varepsilon}}(\sigma, F) \leq M(\sigma, F)
$$


From the assumption of Theorem 7, by (74) and Theorem 4, we can get

$$
\begin{aligned}
\underset{\sigma \rightarrow 0^{+}}{\limsup \frac{X\left(\log ^{+} M_{u}(\sigma, F)\right)}{\log (1 / \sigma)}} & =\underset{\sigma \rightarrow 0^{+}}{\limsup } \frac{X\left(\log ^{+} M_{\widehat{\mathcal{S}}_{\varepsilon}}(\sigma, F)\right)}{\log (1 / \sigma)} \\
& =\underset{\sigma \rightarrow 0^{+}}{\limsup \sin } \frac{X\left(\log ^{+} M(\sigma, F)\right)}{\log (1 / \sigma)}=\rho^{*} .
\end{aligned}
$$

From (75), for any $\varepsilon>0$, we have

$$
\limsup _{\sigma \rightarrow 0^{+}} \frac{X\left(\log ^{+} M\left(\sigma, F, \widehat{S}_{\varepsilon}\right)\right)}{\log (1 / \sigma)}=\rho^{*},
$$

where $M\left(\sigma, F, \widehat{S}_{\varepsilon}\right)=\sup \left\{|F(s)|: \Re(s) \geq \sigma, s \in \widehat{S}_{\varepsilon}\right\}$.

Set $g(z)=F\left((2 \varepsilon / \pi) \Psi(z)+i t_{0}\right)$, where $\Psi(z)$ is stated as in Lemma 16; then, from Lemma 16 , we have that $g(z)$ is analytic in the unit disc $|z|<1$ and satisfies

$$
M\left(\frac{2 \varepsilon}{\pi} \Psi\left(r^{2}\right), F, S_{\varepsilon_{2}}\right) \leq M(r, g) \leq M\left(\frac{2 \varepsilon}{\pi} \Psi(r), F, S_{\varepsilon}\right),
$$

where $0<\varepsilon_{2}<\varepsilon$. Therefore, from the above inequality and Lemma 16, we have

$$
\begin{gathered}
\limsup _{r \rightarrow 1^{-}} \frac{\log ^{+} \log ^{+} M(r, g)}{\log (1 /(1-r))}=\infty, \\
\limsup _{r \rightarrow 1^{-}} \frac{X\left(\log ^{+} M(r, g)\right)}{\log (1 /(1-r))}=\rho^{*} .
\end{gathered}
$$

Then, similar to the proof of Theorem 6 , we can prove that, for any $\eta>0$, the inequality

$$
\limsup _{\sigma \rightarrow 0^{+}} \frac{X\left(\bar{n}\left(\sigma, i t_{0}, \eta, F=a\right)\right)}{\log (1 / \sigma)}=\varrho \geq \rho^{*}
$$

holds for any $a \in \mathbb{C}$, except for one exception, where $\bar{n}\left(\sigma, i t_{0}\right.$, $\eta, F=a)$ is stated as in Theorem 7 .

Thus, we complete the proof of Theorem 7.

\section{Conflict of Interests}

The authors declare that there is no conflict of interests regarding the publication of this paper.

\section{Acknowledgments}

This work was supported by the National Natural Science Foundation of China (11301233 and 61202313), the Natural Science Foundation of Jiangxi Province in China (20132BAB211001), and the Foundation of Education Department of Jiangxi (GJJ14644) of China. The second author is supported in part by Beijing Natural Science Foundation (no. 1132013) and The Project of Construction of Innovative Teams and Teacher Career Development for Universities and Colleges Under Beijing Municipality (CIT and TCD20130513).

\section{References}

[1] M. S. Liu, "The regular growth of Dirichlet series of finite order in the half plane," Journal of Systems Science and Mathematical Sciences, vol. 22, no. 2, pp. 229-238, 2002.

[2] D. C. Sun and J. R. Yu, "On the distribution of values of random Dirichlet series II," Chinese Annals of Mathematics B, vol. 11, no. 1, pp. 33-44, 1990.

[3] J. R. Yu, Dirichlet Series and the Random Dirichlet Series, Science Press, Beijing, China, 1997.

[4] J. R. Yu, "Borel's line of entire functions represented by LaplaceStieltjes transformation," Acta Mathematica Sinica, vol. 13, pp. 471-484, 1963 (Chinese).

[5] C. J. K. Batty, "Tauberian theorems for the Laplace-Stieltjes transform," Transactions of the American Mathematical Society, vol. 322, no. 2, pp. 783-804, 1990.

[6] Y. Y. Kong and D. C. Sun, "On the growth of zero order LaplaceStieltjes transform convergent in the right half-plane," Acta Mathematica Scientia B, vol. 28, no. 2, pp. 431-440, 2008.

[7] Y. Y. Kong and D. C. Sun, "The analytic function in the right halp plane defined by Laplace-Stieltjes transforms," Journal of Mathematical Research \& Exposition, vol. 28, no. 2, pp. 353-358, 2008.

[8] Y. Y. Kong and Y. Hong, On the Growth of Laplace-Stieltjes Transforms and the Singular Direction of Complex Analysis, Jinan University Press, Guangzhou, China, 2010.

[9] Y. Y. Kong and Y. Yang, "On the growth properties of the Laplace-Stieltjes transform," Complex Variables and Elliptic Equations, vol. 59, no. 4, pp. 553-563, 2014.

[10] A. Mishkelyavichyus, "A Tauberian theorem for the LaplaceStieltjes integral and the Dirichlet series," Litovskij Matematicheskij Sbornik, vol. 29, no. 4, pp. 745-753, 1989 (Russian).

[11] L. N. Shang and Z. S. Gao, "Value distribution of analytic functions defined by Laplace-Stieltjes transforms," Acta Mathematica Sinica. Chinese Series, vol. 51, no. 5, pp. 993-1000, 2008.

[12] Y. Y. Kong, "Laplace-Stieltjes transforms of infinite order in the right half-plane," Acta Mathematica Sinica, vol. 55, no. 1, pp. 141148, 2012.

[13] Y. Y. Kong and D. C. Sun, "On type-function and the growth of Laplace-Stieltjes transformations convergent in the right halfplane," Advances in Mathematics, vol. 37, no. 2, pp. 197-205, 2008 (Chinese).

[14] L. N. Shang and Z. S. Gao, "The growth of entire functions of infinite order represented by Laplace-Stieltjes transformation," Acta Mathematica Scientia A, vol. 27, no. 6, pp. 1035-1043, 2007 (Chinese).

[15] P. Wang, "The $P(R)$ type of the Laplace-Stieltjes transform in the right half-plane," Journal of Central China Normal University, vol. 21, no. 1, pp. 17-25, 1987 (Chinese).

[16] P. Wang, "The $P(R)$ order of analytic functions defined by the Laplace-Stieltjes transform," Journal of Mathematics, vol. 8, no. 3, pp. 287-296, 1988 (Chinese).

[17] H. Y. Xu and Z. X. Xuan, "The growth and value distribution of Laplace-Stieltjes transformations with infinite order in the right half-plane," Journal of Inequalities and Applications, vol. 2013, article 273, 15 pages, 2013.

[18] K. Knopp, "Über die Konvergenzabszisse des Laplace-Integrals," Mathematische Zeitschrift, vol. 54, pp. 291-296, 1951. 
[19] T. Cao and H. Yi, "The growth of solutions of linear differential equations with coefficients of iterated order in the unit disc," Journal of Mathematical Analysis and Applications, vol. 319, no. 1, pp. 278-294, 2006.

[20] F. Titzhoff, "Slowly growing functions sharing values," Fizikos ir Matematikos Fakulteto Mokslinio Seminaro Darbai, vol. 8, pp. 143-164, 2005.

[21] H. X. Yi and C. C. Yang, Uniqueness Theory of Meromorphic Functions, Kluwer Academic Publishers, Beijing, China, 2003. 


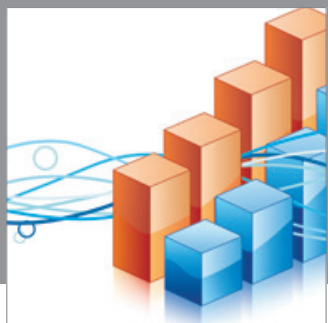

Advances in

Operations Research

mansans

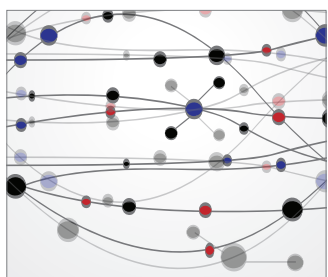

The Scientific World Journal
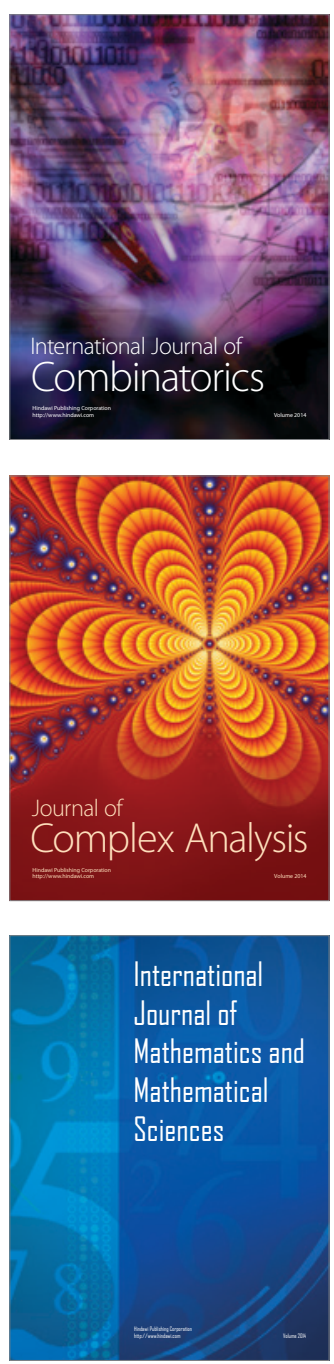
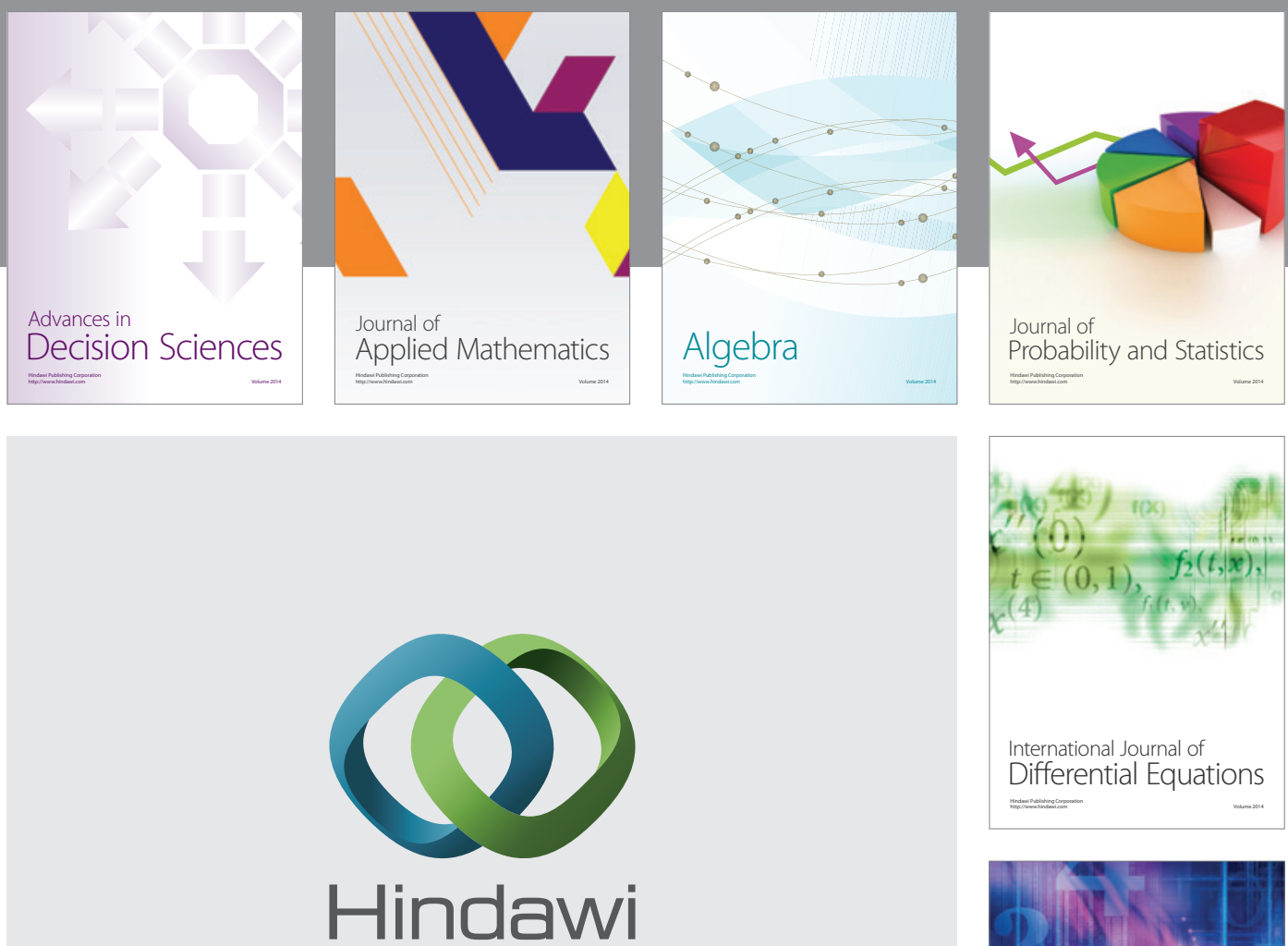

Submit your manuscripts at http://www.hindawi.com
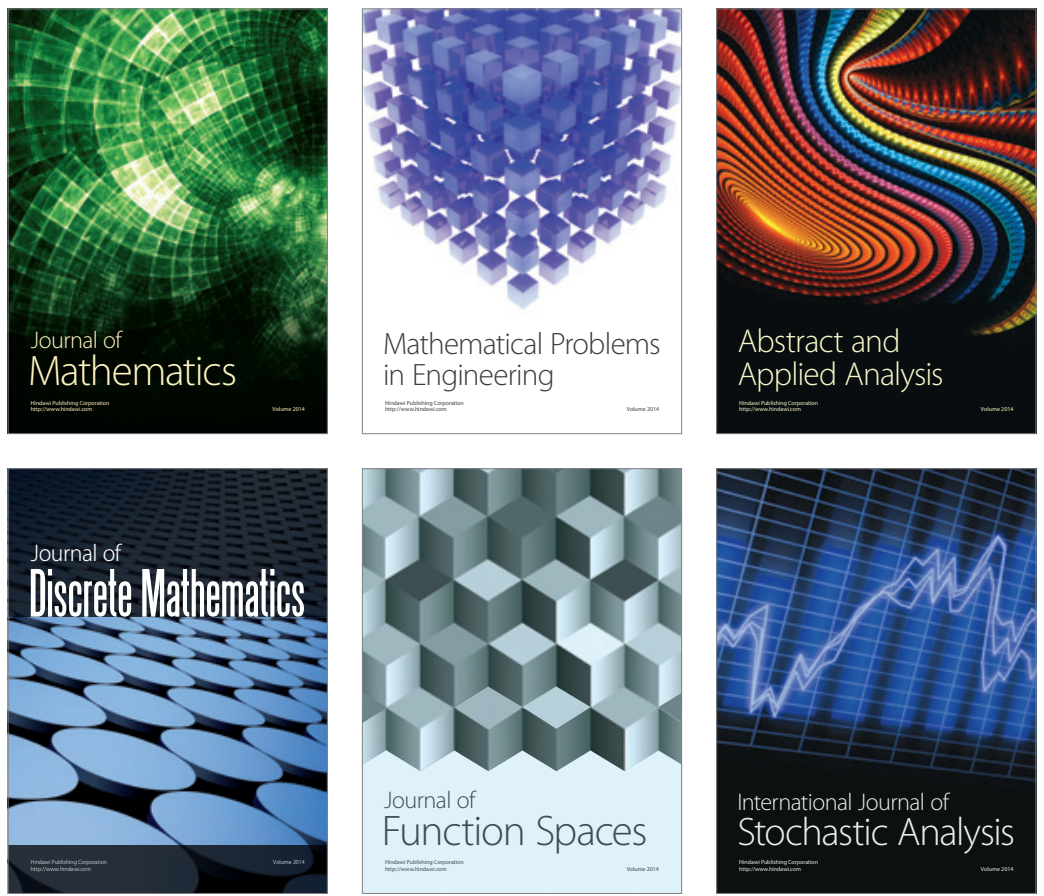

Journal of

Function Spaces

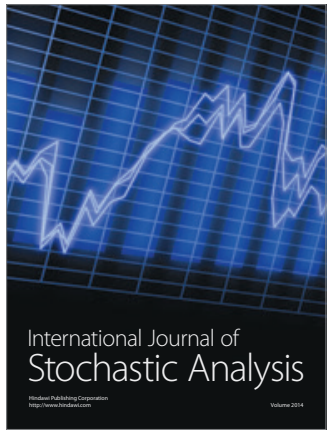

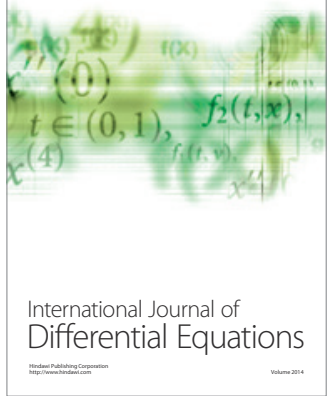
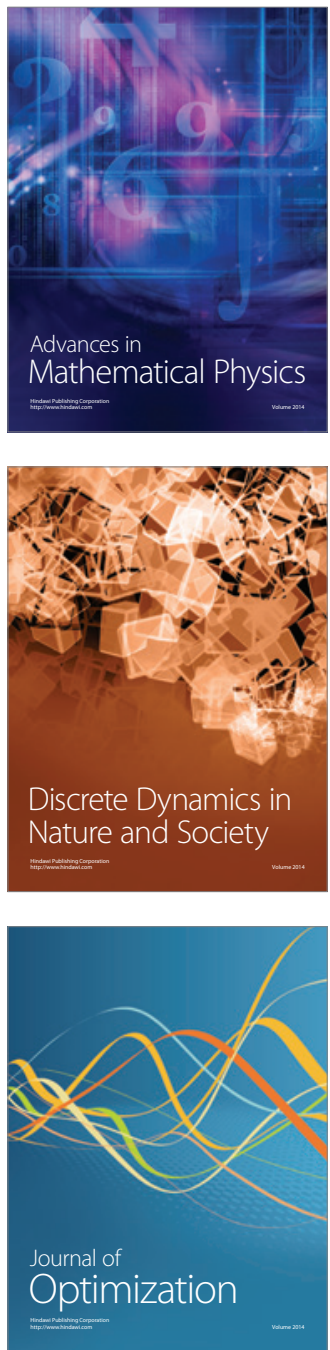